\title{
The masses of hot subdwarfs
}

\author{
X. Zhang ${ }^{1,2}$, X. Chen ${ }^{1}$, and Z. Han $^{1}$ \\ 1 National Astronomical Observatories/Yunnan Observatory, the Chinese Academy of Sciences, Kunming 650011, PR China \\ e-mail: X.F.Zhang@live.com \\ 2 Graduate School of the Chinese Academy of Sciences, PR China
}

Received 16 April 2009 / Accepted 21 August 2009

ABSTRACT

\begin{abstract}
Context. Masses are a fundamental parameter, but they are not well known for most hot subdwarfs. In general, the mass of a hot subdwarf is derived with asteroseismology or dynamical methods, for which it is often difficult to obtain the necessary data from observations.

Aims. We intend to find an approach to deriving the masses of hot subdwarfs from observational data in the literature.

Methods. We presented full evolutionary calculations for hot subdwarfs in a wide mass range $\left(0.33 M_{\odot}\right.$ to $\left.1.4 M_{\odot}\right)$ for a Population I metallicity of $Z=0.02$, and obtained a relation between $M_{\mathrm{p}}$ and $\log \left(\frac{T_{\mathrm{eff}}^{4}}{g}\right)$, where $M_{\mathrm{p}}, T_{\text {eff }}$, and $g$ are the most probable mass, effective temperature, and gravity. This relation is used to study the masses of some observed hot subdwarfs.

Results. We proposed a method of determining the masses of hot subdwarfs. Using this method, we studied the masses of hot subdwarfs from the ESO supernova Ia progenitor survey and Hamburg quasar survey. The study shows that most of subdwarf B stars have masses between 0.42 and $0.54 M_{\odot}$, whilst most sdO stars are in the range $0.40 \sim 0.55 M_{\odot}$. Comparing our study to the theoretical mass distributions of Han et al. (2003, MNRAS, 341, 669), we found that sdO stars with mass less than $\sim 0.5 M_{\odot}$ may evolve from sdB stars, whilst most high-mass $\left(>0.5 M_{\odot}\right)$ sdO stars result from mergers directly.
\end{abstract}

Key words. stars: fundamental parameters - stars: horizontal-branch - subdwarfs

\section{Introduction}

Hot subdwarfs are generally classfied into three types by their spectra. These are subdwarf B ( $\mathrm{sdB}$, with a surface effective temperature, $T_{\text {eff }}$, in a range from 20000 to $40000 \mathrm{~K}$, with H-Balmer absorption lines wider than in normal B stars), subdwarf $\mathrm{O}$ (sdO, $T_{\text {eff }}$ ranging from $40000 \mathrm{~K}$ to $80000 \mathrm{~K}$ with strong He absorption lines), and subdwarf $\mathrm{OB}$ ( $\mathrm{sdOB}$, a transition between $\mathrm{O}$ and B, Moehler et al. 1990; Heber 2009). These objects are located below the upper main sequence on the Hertzsprung-Russell diagram(HRD) and are also known as extreme horizontal branch (EHB) stars from the view of their evolutionary stages; i.e., they are believed to be core He-burning objects with extremely thin hydrogen envelopes $\left(<0.02 M_{\odot}\right)$.

Hot subdwarfs are an important population in several respects. For example, pulsating sdB stars are standard candles in distance determination (Kilkenny et al. 1999). Likewise, close binaries composed of an sdB star and a massive white dwarf (WD) are qualified as supernova Ia progenitors (Maxted et al. 2000). Moreover, hot subdwarfs are an important source of farUV light in the galaxy, and they are successfully used to explain the UV-upturn in elliptical galaxies (Kilkenny et al. 1997; Han et al. 2007).

Several scenarios have been proposed to explain the formation of these objects, i.e. strong mass loss for a star on the red giant branch (Dorman et al. 1993; D’Cruz et al. 1996), mass transfer in close binary systems (Mengel et al. 1976), and the coalescence of two helium white dwarfs (He-WDs) (Iben 1990; Webbink 1984). Recent radial velocity (RV) surveys reveal that two thirds of all sdB stars reside in close binaries (Maxted et al. 2001). Han et al. $(2002,2003)$ propose a binary model for the formation of hot subdwarf stars, in which three channels (stable Roche lobe overflow (RLOF), common envelope (CE) ejection, and merging of two He-WDs) are included. (For details see Han et al. 2002, 2003). This binary model successfully explained most of the observational characteristics of hot subdwarfs, and is now widely used in the study of hot subdwarf stars, e.g. O'Toole et al. (2004).

Mass is a fundamental parameter of stars, but it is very uncertain for most hot subdwarfs, which are assumed to be around $0.5 M_{\odot}$ (Heber 1986; Saffer et al. 1994). Sometimes we need a more precise mass to study these objects further. In recent years, more than 2300 objects have been included in a hand hot subdwarf database (Østensen 2004), among these more than 200 hot subdwarfs have been studied in detail for atmospheric parameters (e.g. Saffer et al. 1994; Maxted et al. 2001; Edelmann et al. 2003; Lisker et al. 2005; Stroeer et al. 2007). However, only a few hot subdwarfs have well-defined masses from asteroseismology, e.g. PG 0014+067 (Brassard et al. 2001), PG 1219+534 (Charpinet et al. 2005a), PG 1325+101(Charpinet et al. 2006), and Feige 48 (Charpinet et al. 2005b). In addition, Wood \& Saffer (1999) show an sdB star HW Vir (PG 1241-082), which is in a double-lined eclipsing binary, to be $0.48 \pm 0.09 M_{\odot}$ from dynamical methods. For other hot subdwarfs, which are not pulsating stars or are in binary systems without detailed light or radial-velocity curves, masses are derived from theoretical evolutionary tracks (Dorman et al. 1993), e.g., choosing the track closest to the data point on the $T_{\text {eff }}-\log (g)$ diagram, where $T_{\text {eff }}$ and $g$ are effective temperature and surface gravity, respectively. The commonly used evolution library of hot subdwarfs is that of Dorman et al. (1993), with masses from 0.4 to $0.5 M_{\odot}$. However, Han et al. $(2002,2003)$ show that the masses of hot subdwarfs 


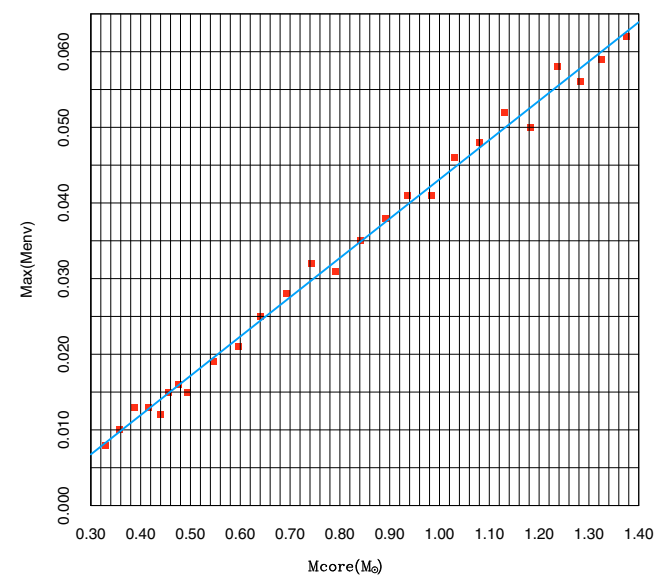

Fig. 1. The maximum envelope masses for different core masses in our hot subdwarf models (squares). The solid line is a linear fitting of the square points, with errors less than $0.001 M_{\odot}$.

can be in a much wider range; i.e., from $0.3 M_{\odot}$ to more than $0.7 M_{\odot}$. Thus, it is necessary to extend the evolution library of hot subdwarfs or find a simple method of estimating the masses of hot subdwarfs.

In this letter, we carry out full evolutionary calculations for hot subdwarfs and obtain an approach to determining the masses of hot subdwarfs. Using this approach, we study the masses and mass distributions of observed hot subdwarfs.

\section{The model}

The stellar evolution code used was originally developed by Eggleton (1971, 1972, 1973). The code has been updated with the latest input physics over the past three decades as described by Han et al. (1994, hereafter HPE) and by Pols et al. (1995, 1998). We set the ratio of mixing length to local pressure scale height, $\alpha=l / H_{\mathrm{p}}$, to 2.0 and the convective overshooting parameter, $\delta_{\text {OV }}$, to 0.12 (Pols et al. 1997; Schröder et al. 2007). The opacity tables for various metallicities are compiled by Chen \& Tout (2007) from Iglesias \& Rogers (1996) and Alexander \& Ferguson (1994). The initial hydrogen mass fraction, $X$, is obtained by, $X=0.76-3.0 \mathrm{Z}$, where $Z$ is the metallicity (Pols et al. 1998).

We constructed a series of zero-age extreme horizontal branch models (ZAEHB, i.e. helium begins stably burning in the core) of Pop I $(Z=0.02)$ and carried out their evolutions in detail. The model grid contains a wide mass range; i.e., the core mass ranges from 0.33 to $1.4 M_{\odot}$, and the envelope mass changes, by a step of about $0.005 M_{\odot}$, from zero to the maximum envelope mass (see Fig. 1), beyond which the constructed object is not a hot subdwarf any more (i.e. $T_{\text {eff }}<20000 \mathrm{~K}$ or $\log (g)<4.5)$.

As an example, we show the evolutionary tracks of hot subdwarfs with a core mass of $0.475 M_{\odot}$ in Fig. 2 . The envelope masses, $M_{\text {env }}$, are $0.000,0.001,0.005,0.010$, and $0.015 M_{\odot}$. TAEHB is the termination-age EHB, i.e., at the core helium exhaustion (Dorman et al. 1993). The region between ZAEHB and TAEHB is known as the main sequence. The age difference between two neighbouring crosses is 10 Myr. From the figure, we see that most of the observed sdB stars are located in the main sequence. However, we cannot confirm their core masses and their evolutionary stages yet, since a star with a different core mass in a different evolutionary stage is also likely in this region of the $T_{\text {eff }}-\log (g)$ diagram (see Sect. 3). For instance, an sdO star is possibly a main sequence star with a core mass higher than that

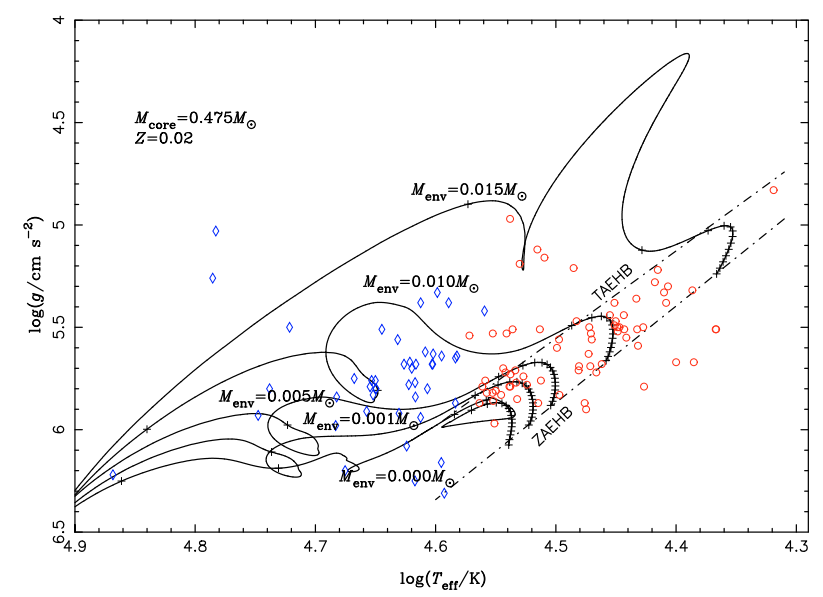

Fig. 2. Evolutionary tracks of hot subdwarfs with a core of $0.475 M_{\odot}$ and $Z=0.02$. The envelope masses are $0.000,0.001,0.005,0.010$, and $0.015 M_{\odot}$. ZAEHB and TAEHB represent the onset and the end of main sequence, respectively (see the text for detail). The circles and diamonds are for sdB and sdO stars, respectively, from the ESO supernova Ia progenitor survey (SPY, Lisker et al. 2005; Stroeer et al. 2007). The age difference between two neighbouring crosses is $10 \mathrm{Myr}$.

of an sdB star, or in the stage of post main sequence of a $\mathrm{sdB}$ star.

\section{Methods}

As we know, more than one evolutionary tracks pass through the same point on the $T_{\text {eff }}-\log (\mathrm{g})$ diagram. Thus, we cannot figure out the most probable one from them just by comparing the evolutionary tracks. To solve this problem, we intend to find another approach to deriving the masses of hot subdwarfs.

Hot subdwarfs are core helium-burning stars with hydrogen envelopes that are too thin to sustain hydrogen burning; therefore, the luminosity of a hot subdwarf, $L$, depends upon the core mass, $M_{\mathrm{c}}$, and the age, $t$. Meanwhile, we know that $L \sim T_{\mathrm{eff}}^{4} \cdot R^{2} \sim M_{\mathrm{c}} \cdot \frac{T_{\mathrm{eff}}^{4}}{g}$, where $R$ is the radius. Thus, we have a relation as $f\left(M_{\mathrm{c}}, t\right) \sim \log \left(\frac{T_{\mathrm{eff}}^{4}}{g}\right)$, where $T_{\text {eff }}$ and $g$ are two fundamental parameters of hot subdwarfs from observations. Thus, we choose $\log \left(\frac{T_{\text {eff }}^{4}}{g}\right)$ as a basic parameter for further study of the masses of these objects.

In general, stars evolves at different speeds in different evolutionary stages. If a star evolves slowly in a certain stage, it has more chance of being observed in this stage. We may then find out the most probable mass via studying the change rate of $\log \left(\frac{T_{\text {eff }}^{4}}{g}\right)$, as described in the following. We divide $\log \left(\frac{T_{\text {eff }}^{4}}{g}\right)$ into small intervals of 0.1 from 10.0 to 16.0 , and calculate the evolutionary time, $\delta t$, of each model in these intervals. Thus, for each interval of $\log \left(\frac{T_{\text {eff }}^{4}}{g}\right)$, we may obtain a core mass, with which a hot subdwarf stays in it for the longest time amongst all the models (i.e. having the maximum value of $\delta t$ ), and has the most chance of being observed. This core mass is then the most probable mass, $M_{\mathrm{p}}$, in the observations, and is assumed to be the real mass of the subdwarf ${ }^{1}$. We finally obtained a relation between $M_{\mathrm{p}}$ and $\log \left(\frac{T_{\mathrm{eff}}^{4}}{g}\right)$, which is shown in Fig. 3. From this relation

\footnotetext{
1 Note that the mass given here is the core mass, which is a lower limit of the total mass of hot subdwarfs.
} 


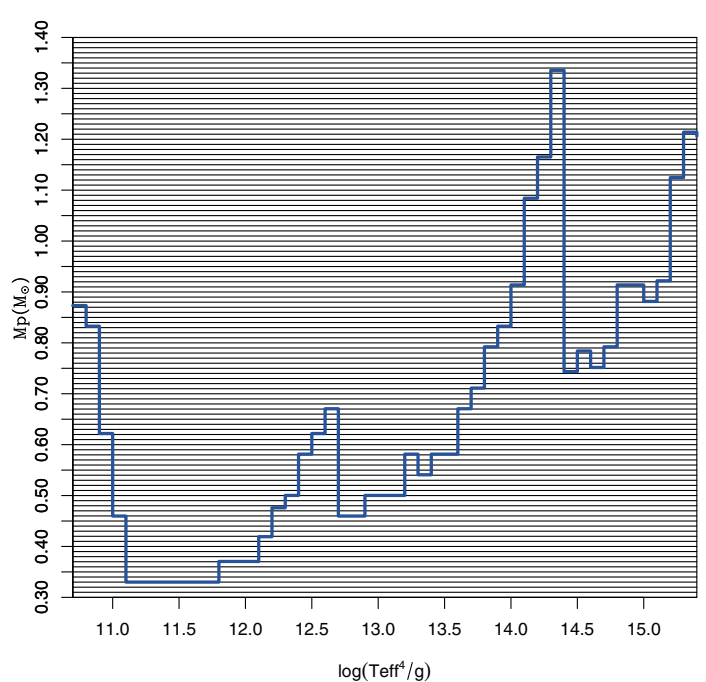

Fig. 3. The $M_{\mathrm{p}}-\log \left(\frac{T_{\mathrm{eff}}^{4}}{g}\right)$ relation. $M_{\mathrm{p}}$ is the most probable mass.

we may simply know the mass of a hot subdwarf for given $T_{\text {eff }}$ and $g$.

Using the $M_{\mathrm{p}}-\log \left(\frac{T_{\text {eff }}^{4}}{g}\right)$ relation, we studied the masses of some observed hot subdwarfs, including $164 \mathrm{sdB}$ stars and $46 \mathrm{sdO}$ stars. Some sdB stars are from the ESO supernova Ia progenitor survey (SPY, Lisker et al. 2005) and the other sdB stars are from the Hamburg quasar survey (HQS, Edelmann et al. 2003). The sdO stars come from SPY (Stroeer et al. 2007). The mass distributions for $\mathrm{sdB}$ and $\mathrm{sdO}$ stars are shown in Figs. 4 and 5 , respectively.

The error on the mass, $\Delta M_{\mathrm{p}}$, depends on the parameter $\log \left(\frac{T_{\mathrm{eff}}^{4}}{g}\right)$, and this dependence is shown in the upper panel of Fig. 6, where $f\left(\equiv \frac{\Delta M_{\mathrm{p}}}{\Delta \log \left(T_{\text {eff }}^{4} / g\right)}\right)$ is derived from the $M_{\mathrm{p}}-\log \left(\frac{T_{\text {eff }}^{4}}{g}\right)$ relation. The $\log \left(\frac{T_{\text {eff }}^{4}}{g}\right)$ distributions of $\mathrm{sdB}$ and $\mathrm{sdO}$ stars from the SPY and the HQS are shown in the bottom panel of this figure. From this figure we see that $f$ is lower than 0.5 for most hot subdwarfs. To discuss the error on the mass obtained from our method, we should consider the error from both theory and observation. The $M_{\mathrm{p}}-\log \left(\frac{T_{\text {eff }}^{4}}{g}\right)$ relation here is derived from Pop I models $(Z=0.02)$. Thus, the different metalicities result in a different $\log \left(\frac{T_{\text {eff }}^{4}}{g}\right)$ for the same mass. For example, $\Delta \log \left(\frac{T_{\text {eff }}^{4}}{g}\right)$ is about 0.04 dex for the models between $Z=0.0001$ and $Z=0.06$. For stars from the ESO supernova Ia progenitor survey, the observational errors for sdB stars are $\Delta T_{\text {eff }}=360 \mathrm{~K}$ and $\Delta \log (\mathrm{g})=0.05 \mathrm{dex}$, and the observational errors for $\mathrm{sdO}$ stars are $\Delta \log \left(T_{\text {eff }}\right)=0.011 \mathrm{dex}$ and $\Delta \log (\mathrm{g})=0.097 \mathrm{dex}$, respectively. These correspond to $\Delta \log \left(T_{\text {eff }}^{4} / g\right)$ of 0.07 dex and 0.14 dex. Thus, the total errors, $\Delta \log \left(T_{\text {eff }}^{4} / g\right)$ (theory plus observation), are $0.11 \mathrm{dex}$ and $0.18 \mathrm{dex}$, respectively. According to the $f \sim \log \left(\frac{\mathrm{T}_{\text {eff }}^{4}}{\mathrm{~g}}\right)$ relation shown in Fig. 6, the errors on the masses are less than $0.055 M_{\odot}$ and $0.09 M_{\odot}$ for most sdB and sdO stars, respectively. For sdB stars from the Hamburg Quasar Survey, we derived the mass errors for each star in a similar way. The results show that the majority of errors are in the $0.097 M_{\odot}$ to $0.117 M_{\odot}$ range (95 percent confidence interval), while the mean error is $0.107 M_{\odot}$.
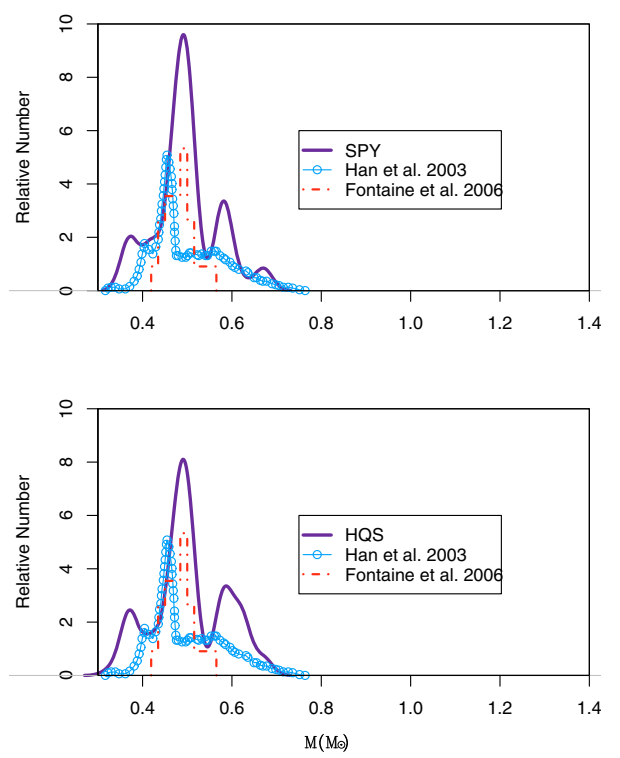

Fig. 4. The mass distribution of sdB stars (derived from the $M_{\mathrm{p}}-\log \left(\frac{T_{\text {eff }}^{4}}{g}\right)$ relation) from the ESO supernova Ia progenitor survey (SPY, Lisker et al. 2005) and the Hamburg quasar survey (HQS, Edelmann et al. 2003). As a comparison, we also show the mass distribution from the theoretical study of Han et al. (2003) and from asteroseismology (Fontaine et al. 2006).

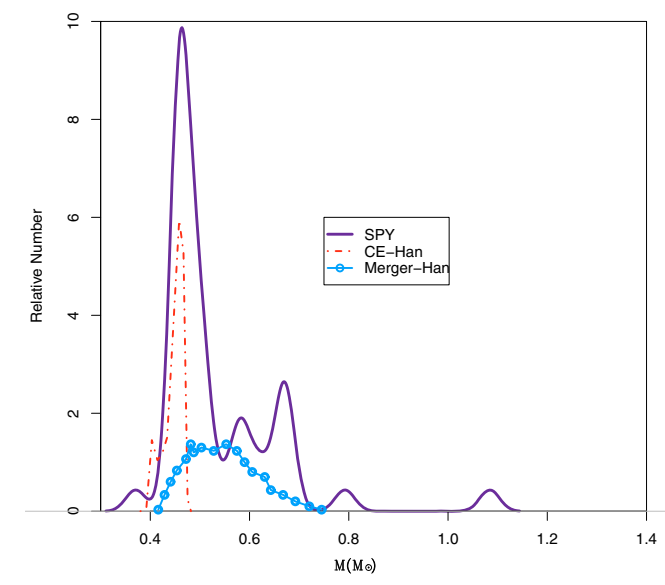

Fig. 5. The mass distribution of $\mathrm{sdO}$ stars (derived from the $M_{\mathrm{p}^{-}}$ $\log \left(\frac{T_{\text {eff }}^{4}}{g}\right)$ relation) from the ESO supernova Ia progenitor survey (SPY, Lisker et al. 2005). The mass distributions from the CE and merger scenarios (Han et al. 2003) $)^{2}$ are also shown in this figure.

\section{Results and discussion}

As shown in Fig. 4, the sdB stars from SPY and HQS have the same mass distributions - the KS test shows that the two samples come from the same distribution at a high level of confidence $(>93.5 \%)$. Most of the $\mathrm{sdB}$ stars have masses ranging from 0.42 to $0.54 M_{\odot}$, and the mean mass is about $0.50 M_{\odot}$, equal to that assumed in previous studies. As a comparison, we also show the mass distributions from the theoretical study of Han et al. $(2003)^{2}$ and from asteroseismology (Fontaine et al. 2006) in Fig. 4, from which we see that the distribution obtained in this paper matches the other two closely.

\footnotetext{
2 In the paper of Han et al. (2003), the mass distribution are for both $\mathrm{sdB}$ and sdO stars. We seperated these objects, thus the distribution presented here is different from that of Han et al. (2003).
} 


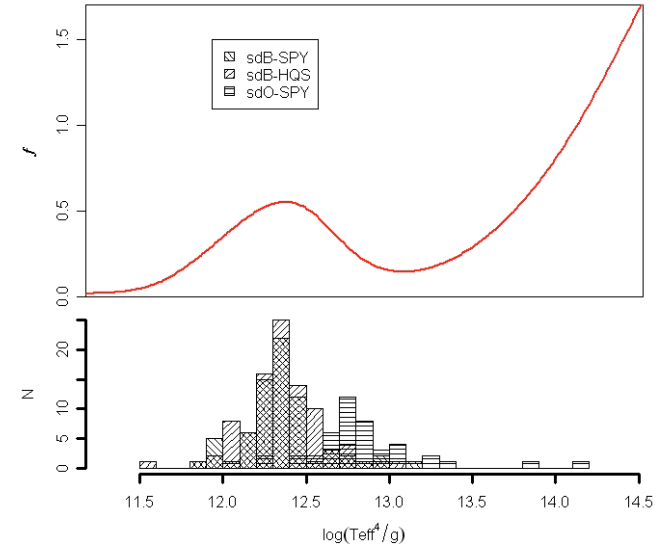

Fig. 6. The upper panel: the dependence of the mass errors on $\log \left(\frac{\mathrm{T}_{\mathrm{eff}}^{4}}{\mathrm{~g}}\right)$, derived from the $M_{\mathrm{p}}-\log \left(\frac{T_{\text {eff }}^{4}}{g}\right)$ relation, where $f \equiv \frac{\Delta M_{\mathrm{p}}}{\Delta \log \left(T_{\text {eff }}^{4} / g\right)}$. The bottom panel: the $\log \left(\frac{T_{\text {eff }}^{4}}{g}\right)$ distributions of $\mathrm{sdB}$ and $\mathrm{sdO}$ stars from the SPY and the HQS.

Figure 5 shows that most sdO stars are in $0.40 \sim 0.55 M_{\odot}$, although some sdO stars have much higher masses than these values. Since low-mass hot subdwarfs (e.g. less than $\sim 0.5 M_{\odot}$ ) can not reach $40000 \mathrm{~K}$ (the minimum temperature for sdO stars) during the main sequence (see Fig. 2 in Han et al. 2002), sdO stars with mass less than $\sim 0.5 M_{\odot}$ most likely evolve from sdB stars, i.e., these objects initially appear as sdB stars when they are on the main sequence, and as sdO stars when they evolved off the main sequence (post-EHB). The typical evolutionary time scales for hot subdwarfs on the MS and post-EHB are $\sim 160$ and $\sim 20 \mathrm{Myr}$, respectively. Thus, observationally the number of sdO stars (evolving from sdB stars) is expected to be about $12 \%$ of that of sdB stars, and for SPY this equates to about 9 objects. They are likely He-deficient sdO stars. There are $13 \mathrm{He}$-deficient sdO stars in SPY, and 7 ones (likely evolve from sdB stars) of them with masses less than $\sim 0.5 M_{\odot}$ in our study, similar to the 9 predicted by theory.

To more easily compare with theoretical results, we presented the mass distributions of sdO stars obtained from Han et al. (2003) ${ }^{2}$ in Fig. 5, where the dot-dashed line is from CE ejection and the circle-line is from the merger of two He-WDs. We see that $\mathrm{CE}$ ejection can only account for sdO stars with masses lower than $\sim 0.5 M_{\odot}$. The He-He merger mainly contributes to high-mass sdO stars, but it is also responsible for a small fraction of low-mass sdO stars. In particular, the fraction of very low-mass stars $\left(<0.4 M_{\odot}\right)$ is smaller among sdO stars than among sdB stars, as shown in Figs. 4 and 5. This difference probably stems from very few sdB stars with masses lower than $0.4 M_{\odot}$ being hotter than $40000 \mathrm{~K}$ during their post-EHB stage.

We know that a binary resulting from $\mathrm{CE}$ ejection generally has a short orbital period. Thus, if low-mass sdO stars are mainly from the CE ejection channel, then a large fraction of these objects should be observed in short orbital period binaries. Among $23 \mathrm{sdO}$ stars with masses less than $\sim 0.5 M_{\odot}$, (if they indeed evolve from $\mathrm{sdB}$ stars), we would expect a fraction of binaries similar to that of sdB stars, i.e. 39\% (Napiwotzki et al. 2004). However, only five sdO stars have been identified in binaries from SPY. The possible reasons for the low fraction are (a) the He-He merger contributes a small fraction of low-mass sdO stars, which are all singles; (b) low-mass sdO stars from
CE ejection generally have lower mass companions (most likely M, see Fig. 15 in Han et al. 2003), and may be identified as singles; (c) some other observational effects, such as random inclination of orbital planes, contribute to a decrease in detection efficiency. Moreover, in comparison to the merger channel, $\mathrm{CE}$ ejection, which may leave a thin hydrogen-rich envelope after the ejection, often produces hot subdwarfs with surface helium deficiency. Thus, if sdO stars are identified in binaries with short orbital periods, they are most likely to be helium deficient, since the merger of He-He WDs can only produce single heliumenriched sdO stars. The observations confirm this prediction; i.e., four of the five identified binary sdO stars have short orbital periods and are helium deficient. An extreme case from the $\mathrm{CE}$ ejection channel is that all of the common envelope has been ejected, leading to a naked He core (hot subdwarfs) and a companion. This may produce helium-enriched sdO stars with short orbital periods. Since this case is rare, the fraction of helium enriched sdO binaries among $\mathrm{He}-\mathrm{sdO}$ stars (most of them are from the merger channel) is very low (4\% at most, Napiwotzki et al. 2004, see also Heber 2008).

Acknowledgements. We thank an anonymous referee for his/her valuable comments that helped us to improve the paper. X.Z. thanks Dr. Richard Pokorny for improving the English language of the original manuscript. This work was in part supported by the Natural Science Foundation of China under Grant Nos. 10821061,10603013, and 2007CB815406, the Chinese Academy of Science under Grant Nos. 06YQ011001, and the Yunnan National Science Foundation (Grant No. 08YJ041001).

\section{References}

Alexander, D. R., \& Ferguson, J. W. 1994, ApJ, 437, 879

Brassard, P., Fontaine, G., Billères, M., et al. 2001, ApJ, 563, 1013

Charpinet, S., Fontaine, G., Brassard, P., et al. 2005a, A\&A, 437, 575

Charpinet, S., Fontaine, G., Brassard, P., et al. 2005b, A\&A, 443, 251

Charpinet, S., Silvotti, R., Bonanno, A., et al. 2006, A\&A, 459, 565

Chen, X., \& Tout, C. A. 2007, Chin. J. Astron. Astrophys., 7, 245

Dorman, B., Rood, R. T., \& O'Connell, R. W. 1993, ApJ, 419, 596

D'Cruz, N. L., Dorman, B., Rood, R. T., et al. 1996, ApJ, 466, 359

Edelmann, H., Heber, U., Hagen, H. -J., et al. 2003, A\&A, 400, 939

Eggleton, P. P. 1971, MNRAS, 151, 351

Eggleton, P. P. 1972, MNRAS, 156, 361

Eggleton, P. P. 1973, MNRAS, 163, 179

Fontaine, G., Brassard, P., Charpinet, S., et al. 2006, ESA SP, 624, 32

Heber, U. 1986, A\&A, 155, 33

Heber, U. 2008, ASPC, 391, 245

Heber, U. 2009, ARAA, 47, 211

Han, Z., Podsiadlowski, Ph., \& Eggleton, P. P. 1994, MNRAS, 270, 121 (HPE)

Han, Z., Podsiadlowski, P., Maxted, P. F. L., et al. 2002, MNRAS, 336, 449

Han, Z., Podsiadlowski, P., Maxted, P. F. L., et al. 2003, MNRAS, 341, 669

Han, Z., Podsiadlowski, Ph., \& Lynas-Gray, A. E. 2007, MNRAS, 380, 1098

Iben, I. J. 1990, ApJ, 353, 215

Iglesias, C. A., \& Rogers, F. J. 1996, ApJ, 464, 943

Kilkenny, D., Koen, C., O’Donoghue, D., et al. 1997, MNRAS, 285, 640

Kilkenny, D., Koen, C., Jeffery, J., et al. 1999, MNRAS, 310, 1119

Lisker, T., Heber, U., Napiwotzki, R., et al. 2005, A\&A, 430, 223

Mengel, J. G., Norris, J., \& Gross, P. G. 1976, ApJ, 204, 488

Maxted, P. F. L., Marsh, T. R., \& North, R. C. 2000, MNRAS, 317, L41

Maxted, P. F. L., Herber, U., Marsh, T. R., et al. 2001, MNRAS, 326, 1391

Meohler, S., Richtler, T., de Boer, K. S., et al. 1990, A\&AS, 86, 53

Napiwotzki, R., Karl, C. A., Lisker, T. et al. 2004, Ap\&SS, 291, 321

O’Toole, S. J., Heber, U., \& Benjamin, R. A. 2004, A\&A, 422, 1053

Pols, O. R., Tout, C. A., Eggleton, P. P, et al. 1995, MNRAS, 274, 964

Pols, O. R., Tout, C. A., Schröder, K. P., et al. 1997, MNRAS, 289, 869

Pols, O. R., Schroder, K.-P., Hurley, J. R., et al. 1998, MNRAS, 298, 525

Saffer, R. A., Bergeron, P., Koester, D., et al. 1994, ApJ, 432, 351

Schröder, K. P., Pols, O. R., \& Eggleton, P. P. 1997, MNRAS, 285, 696

Stroeer, A., Heber, U., Lisker, T., et al. 2007, A\&A, 462, 269

Webbink, R. F. 1984, ApJ, 277, 355

Wood, J. H., \& Saffer, R. 1999, MNRAS, 305, 820

Østensen, R. H. 2004, Ap\&SS, 291, 263 